\title{
A Service Evaluation Exploring The Factors Paramedics Consider When Selecting a Conveyance Destination For Survivors of Medical Out-Of- Hospital Cardiac Arrest.
}

Oliver Zorab ( $\square$ ollie.zorab2@swast.nhs.uk)

South Western Ambulance Service NHS Foundation Trust https://orcid.org/0000-0002-8838-6782

Kim Kirby

South Western Ambulance Service NHS Trust: South Western Ambulance Service NHS Foundation Trust, South Western Ambulance Service NHS Foundation Trust

Matthew Griggs

Plymouth University: University of Plymouth

Original research

Keywords: Out-of-hospital cardiac arrest, paramedic, decision making.

Posted Date: September 3rd, 2021

DOl: https://doi.org/10.21203/rs.3.rs-847311/v1

License: (c) (1) This work is licensed under a Creative Commons Attribution 4.0 International License.

Read Full License 


\section{Abstract \\ Background}

Current guidance suggests that adult survivors of medical out-of-hospital cardiac arrest (OHCA) should be considered for direct transport to specialist centres. Much of the research that informs this recommendation originates from urban environments where patients achieve rapid access to hospital. The factors which facilitate this transport model remain unclear. This regional service evaluation sought to explore the factors influencing paramedic decision making when selection a conveyance destination for survivors of medical OHCA with sustained spontaneous circulation in rural South West England.

\section{Methods}

Qualitative data was collected from two socially-distanced focus groups lead by a facilitator using a predetermined topic guide. Thematic analysis was subsequently used to explore the factors influencing paramedic decision-making when selecting a destination for survivors of medical OHCA. Nine paramedic participants took part in the evaluation from a single regional UK ambulance service.

\section{Findings}

: Factors considered by participants were categorised under five primary themes; published guidance, hospital characteristics, non-technical skills, patient characteristics and technical skills. Subthemes were identified resonating with existing literature including proximity to hospital, access to specialist services and the availability of enhanced prehospital care. Additional factors including dealing with conflict, decision making based on experience and a reliance on local guidance emerged and were felt to be unexpected.

\section{Conclusion}

Paramedics consider multiple factors when selecting a destination for survivors of OHCA, including the hospital's distance from scene and access to specialist services. The patient's stability and availability of enhanced prehospital care to manage agitation are also considered. Paramedics mainly use local guidance, or rely on previous experience to help support their decision making and may need to overcome conflict from hospital colleagues to select an appropriate conveyance destination.

\section{Background}

Each year in the United Kingdom (UK) approximately 30,000 patients receive a resuscitation attempt by Emergency Medical Services (EMS) after suffering an out of hospital cardiac arrest (OHCA) [1]; including 
around 3,000 patients in South West England. Survival from OHCA remains poor with less than $10 \%$ of patients being discharged from hospital alive [2].

A variety of medical aetiologies can result in OHCA; however, coronary heart disease is regarded as the most common cause of cardiac arrest in adults [3]. Following OHCA, achieving an early return of spontaneous circulation (ROSC) is a key predictor of good neurological outcome [4]. Once ROSC has been achieved, clinicians can use guidance from the Resuscitation Council UK (RCUK) to support decision making on topics including airway management and haemodynamic support [5]. Despite this national guidance, there is still variation in survival between hospitals with some evidence indicating an association between increased survival and treatment at a specialist cardiac arrest centre (CAC) for certain patients [6] including those with a presumed cardiac cause.

Although inconsistencies exist between definitions of a CAC within the literature [7], various studies suggest that transferring selected patients directly from an OHCA scene to centres with access to highlevel services such as primary percutaneous coronary intervention (PPCl) may improve survival $[8,9]$. Much of the work exploring this subject has originated from urban metropolitan centres where OHCA patients achieve quicker access from EMS systems and there are reduced journey times to specialist facilities [10] [11]. Nevertheless, current guidance suggests that eligible patients should be considered for direct conveyance to specialist care, however, practical details on how local EMS systems implement this recommendation remain unclear. There is a paucity of data that adequately describes the factors that affect paramedic decision making when conveying survivors of OHCA for onward care. This lack of available information could hamper service development by failing to identify pertinent barriers and enablers which facilitate the commissioning of direct transfer pathways for OHCA patients to specialist centres.

This service evaluation was developed to explore current local paramedic practice in a predominantly rural environment and to identify factors which affect the conveyance of patients with sustained ROSC directly to specialist centres following a medical OHCA.

\section{Methods}

\section{Aim}

This service evaluation aimed to use a phenomenological qualitative method of inquiry to explore the factors paramedics consider when selecting a conveyance destination for OHCA patients with ROSC. The project had the following objectives:

1. To describe the experiences and opinions of paramedics who have cared for critically unwell patients with sustained spontaneous circulation following a medical OHCA in rural South West England.

2. To determine the factors which constrain and enable the conveyance of OHCA patients with ROSC directly to a specialist cardiac centre from a rural area. 


\section{Design}

The evaluation collected qualitative data utilising small online focus groups to understand current practice within the regional NHS ambulance service. This approach allowed participants to remain socially distant, in consideration of the ongoing COVID-19 pandemic. Collected qualitative data was subsequently coded and analysed using thematic analysis (TA).

\section{Setting}

Paramedics employed by the predominantly rural regional ambulance service were eligible to take part in the project. The evaluation used purposive sampling to ensure that only clinicians who had exposure to a recent OHCA incident and, in particular, to a patient with ROSC could participate.

The project utilised the following exclusion and inclusion criteria:

\begin{tabular}{|ll|}
\hline Inclusion & Exclusion \\
\hline - HCPC registered paramedic & - Any non-HCPC employees \\
- Current NHS Trust employee & - Non- NHS Trust employees \\
$\begin{array}{l}\text { - Clinician has attended a patient with spontaneous } \\
\text { circulation (ROSC) following and OHCA in the last 12 } \\
\text { months. }\end{array}$ & - Clinicians who have NOT attended an \\
\hline
\end{tabular}

Paramedics employed by a single regional ambulance service were invited to participate in the project. Email initiations were sent to all 1,780 paramedic employees regardless of recent operational experience. Additional advertisements were circulated in internal organisational communications. Potential participants contacted the project lead to express an interest in participation and were provided with further information. Eligibility to participate and consent was confirmed prior to the beginning of the focus groups.

\section{Procedure}

A topic guide was created to support conversations between the facilitator and project participants. By extrapolating topics explored in existing literature, a guide was developed to provide structure to focus group discussions. The draft topic guide was created and refined following a pilot focus group held with two clinicians who were unfamiliar with the project.

Each online focus group was audio recorded and stored securely on a password protected computer. Following initial introductions, participants were reminded of patient confidentiality before being asked to comment on a fictitious OHCA case. This acted as an icebreaker encouraging comments between all participants. By discussing the fictitious case, participants were able to begin conversation with little further prompting from the facilitator. 
Once conversation between participants had started, input from the facilitator was reduced so not to influence the discussion [12]. Throughout the focus group, the topic guide was used by the facilitator to ensure that necessary areas of interest had been covered, whilst encouraging contribution from all participants. Both focus groups concluded within one hour.

A total of twelve individuals responded to the recruitment emails and advertisements expressing an interest in the project. All twelve volunteers initially agreed to attend one of two focus groups split over two days. Both groups experienced participants who did not attend; resulting in a total of nine participants split across the two focus groups (FG1 - four participants, FG2 - five participants).

\section{Data Analysis}

Following data collection, a seven stage approach [12] was used to thematically analyse the focus group conversations. Recordings were initially transcribed and cleansed by removing names of local hospitals and ambulance services. The data was subsequently reviewed to identify comments relevant to the evaluation's objectives. Codes were applied to comments from both data transcripts before being grouped into primary and secondary themes with a thematic map created. To help increase confidence in the project's data analysis process and conclusions, a further independent review was conducted by a research paramedic employed by the regional ambulance service.

\section{Results}

Following completion of transcript coding, themes were identified and grouped under five primary central themes. A further twelves secondary themes were identified demonstrated in Fig. 1

\section{Guidance}

During both focus groups participants commented on the guidance used when caring for OHCA patients with ROSC. This guidance ranged from national and local publications to information received from faceto-face training. In addition to this, the usefulness of local ambulance service guidance was emphasised in comments made from multiple participants in both groups. In particular, participants felt that the local ambulance service's own guidance was useful whilst delivering care for patients with ROSC.

\section{"The guidance is very clear, very simple and very easy to remember" - FG2P1}

In addition to local guidance, participants described some of the national publications which were considered when selecting a conveyance destination for OHCA patients, in particular, comments relating to guidelines from RCUK were found in the transcripts. Face-to-face training was felt by multiple participants to have been the most valuable in helping them manage patients with ROSC. However, despite recent improvements in the training provided to local paramedics, participants noted that additional guidance could be made available to support their decision-making, in particular patient selection for immediate transfer to a specialist centre. 
"There isn't any guidance that's clear on determining a cardiac cause" - FG2P3

\section{Hospital Characteristics}

The characteristics of the receiving hospitals selected by paramedics to receive OHCA patients were discussed widely in both focus groups. Comments relating to the hospital's distance and access to specialist services were identified from the transcripts from both groups. In addition to describing the physical distance to the hospital, comments were made regarding the time taken to reach receiving centres.

I figure out the quickest route to get to a hospital that's able to receive the patient" - FG1P1

The operating hours and availability of specialist services, in particular $\mathrm{PCl}$, was also discussed by participants as a factor influencing the selection of a conveyance destination. However, the groups elicited more nuanced factors including access to pre-alert telephone numbers and equipment compatibility which were also considered in their destination selection.

"When you're trying to find the phone number for a hospital you don't know - it's stressful - FG1P3

The concept of a hospital's catchment area or "patch" was also widely discussed by participants, in particular when attending cases crossing usual operational boundaries. The conversation in focus group one continued to explore the issue further with participants suggesting conveyance was more likely to occur when a receiving facility is more familiar with an individual clinician.

"I know it's out of the < Hospital > catchment area, but I'm comfortable there" - FG1P1

\section{Non-Technical Skills}

Conversation relating to the impact of non-technical skills during the decision making process was discussed by participants with codes triangulated from both groups. Non-technical skills are commonly defined as cognitive and interpersonal aspects of delivering a safe service [13]. In the context of healthcare, this can range from social skills (e.g. communication and teamwork) to abilities such as interprofessional behaviours [13]. Notably, the concept of conflict was frequently mentioned by participants from both groups; the source of this conflict varied and included interactions with both in-hospital and pre-hospital colleagues.

"I did a case where neither hospital wanted the patient, hospital politics I think is definitely a factor" FG2P1

"There would be quite a backlash if you turn up at < Hospital > instead of < Hospital>" - FG1P2

The professional identity of the pre-hospital clinician providing the pre-alert message to hospitals was discussed by participants as one potential barrier which impacted on their ability to access specialist care. 
In addition, participants reflected on their own previous experiences of managing OHCA patients. Comments were made relating to the knowledge gained through previous experience and how this heavily influenced an individual's ability to make future conveyance decisions.

\section{Patient Characteristics}

Comments relating to a patient's condition were triangulated from both focus groups; in particular managing agitation was discussed. Participants often referred to a patient's agitated state as a key factor impacting on their destination selection. Patient irritability and agitation is a well-documented sequelae following ROSC and should be managed to promote cardiovascular stability and improved oxygenation and ventilation [14] In addition to agitation, both groups included discussion on broader topics including considering a patient's overall level of consciousness as a factor which impacts hospital selection.

With agitated patients, they can pull out IV lines, you can't look after them if you're trying to keep them still" - FG1P4

Finally, establishing and promoting haemodynamic stability was a concept discussed during the project as a key factor considered by paramedics when considering the distance or time they would be willing to travel to reach specialist care.

"I think the more deeply unconscious they are, the easier they are to handle" FG1P1

"As long as they're stable, l'd much rather go to the most suitable hospital" - FG1P4

\section{Technical Skills}

This theme related to comments made by participants regarding the technical or clinical skills required to manage patient in the immediate phase of care following a ROSC. Participants often linked the clinical skills required to the condition of the patient they were treating, for instance, participants commented on how the presence of teams with the ability to sedate or anaesthetise could positively influence their choice of destination. Finally, in addition to agitation, multiple participants discussed the clinical skills required to achieve airway protection or cardiovascular stability as one indicator for deciding which hospital to convey a patients

"Will they need a sedation or anaesthetic beforehand?" - FG1P2

"The anaesthetic was a godsend, just settled him down so he could be managed" - FG2P1

\section{Discussion}

Many of the themes identified in this evaluation resonate with variables described in existing literature and could therefore be broadly considered as expected findings. For instance, the distance between the scene of an incident and a receiving hospital has been explored in various studies $[15,16,17]$ and 
correlates with comments from participants in both focus groups. Participants expressed this phenomenon both in the context of time (i.e. how quickly a patient could get to hospital) and distance (e.g. conveying to the nearest hospital) suggesting that either factor could influence the decision making. Furthermore, participants shared their experiences of conveying OHCA patients over long distances, with journey times of one hour expressed by a participant, compared to shorter distances such as 2.4 miles [17] or 14 mins [15] found in studies from urban areas. This further strengthens the opinion that the direct conveyance of OHCA patients to specialist centres may not be generalisable to rural practice in South West England.

Conveying patients to centres with access to specialist services was a topic discussed by participants. Although much of the existing evidence base describes a multitude of desirable in-hospital services such as $\mathrm{PPCl}$ or comprehensive intensive care [6] [18] focus group participants often only referred to access to $\mathrm{PPCl}$ as a specialist intervention desirable following OHCA

The abilities of on-scene clinicians was debated in focus groups with participants sharing positive experiences of managing patients with the support of prehospital critical care teams (CCTs) capable of delivering sedation and anaesthesia, in particular to manage patient agitation. Clinicians from both focus groups suggested that the presence of these teams could positively facilitate the conveyance of certain patients for onward specialist care. The presence of CCTs at OHCA is widely described [19] [20], however, the cost-effectiveness of this deployment model has recently been questioned due to the poor association between CCT attendance and survival [21, 22].

Finally, the project elicited comments relating to the guidance used by participants to select a conveyance destination following OHCA. Whilst the concept of paramedics seeking evidence to support their decision is a core standard of proficiency for registered clinicians [23], the main source of this guidance was uncertain in this context. Participants commented on a range of sources, including national algorithms, however, the majority of comments in this theme related to locally delivered face-to-face training and service guidelines. However, the use of past experience to help guide decision making was also discussed by participants with comments from both focus groups suggesting that conveyance destination selection was based on previous positive encounters and local familiarity. Comments made by participants relating to overcoming inter-professional conflict as a factor influencing the selection of a conveyance destination for patients were considered unexpected.

\section{Limitations}

This service evaluation was not designed to understand practice outside of the regional ambulance service, therefore the study's findings are not generalizable outside of this context. In addition, the project's limited data collection strategy, developed during the global pandemic, may have further reduced the reliability of the conclusions. The focus group facilitator's role as a paramedic with a CCT deployed by the regional ambulance service could have biased participants to including this service within their discussion. 


\section{Conclusion}

This service evaluation used qualitative inquiry to explore the factors influencing paramedic decision making when selecting a conveyance destination for patients with sustained ROSC following OHCA. Although not generalisable outside of the regional ambulance service, the findings of the project suggest that local paramedics consider a range of factors when selecting a conveyance destination for survivors of OHCA, including the distance from scene and access to PPCl services. The stability of the patient and availability of additional clinical resources to manage agitation are also considered. Paramedics predominantly turn to local guidance, or rely on previous experience to help support their decision making and may need to overcome conflict from hospital colleagues to select an appropriate conveyance destination for patients.

The findings of the evaluation will be used to further enhance local paramedic OHCA training, improve inter-professional working and to inform the development of robust local guidelines to further support ambulance clinicians select destinations for patients following OHCA. In addition, the project has highlighted the need to research and develop cost effective strategies to support the management of post-resuscitation agitation to help facilitate the conveyance of patients following OHCA to specialist care.

\section{List Of Abbreviations}

\begin{tabular}{|ll|}
\hline CAC & Cardiac Arrest Centre \\
\hline CCT & Prehospital Critical Care Team \\
\hline EMS & Emergency Medical Services \\
\hline OHCA & Out of Hospital Cardiac Arrest \\
\hline PPCI & Primary Percutaneous Coronary Intervention \\
\hline RCUK & Resuscitation Council (UK) \\
\hline TTM & Targeted Temperature Management \\
\hline UK & United Kingdom \\
\hline
\end{tabular}

\section{Declarations}

\section{Ethical Approval and Consent to Participate}

Ethical approval for this service evaluation was given by the University of Plymouth's Faculty of Health Research Ethics and Integrity Student Committee on $17^{\text {th }}$ July 2020. In addition, the project was reviewed by South Western Ambulance Service NHS Foundation Trust's Research and Development group and was 
granted approval on $22^{\text {nd }}$ April 2020. Study participants received a Participant Information Sheet prior to the focus group and provided consent to participate.

\section{Consent for Publication}

Not Applicable

\section{Availability of Data and Materials}

The dataset used and analysed during the project are available from the corresponding author on reasonable request.

\section{Competing Interests}

The authors declare that they have no competing interests.

\section{Funding}

None Declared

\section{Authors' Contributions}

$\mathrm{OZ}$ devised the project, collected qualitative data, analysed the findings and prepared the manuscript under supervision from MG. KK provided critical feedback and comments on the final manuscript.

\section{Acknowledgements}

The authors wish to acknowledge the contribution of the paramedic participants who gave their time generously to take part in this evaluation despite the ongoing additional pressures caused by the COVID19 pandemic.

\section{Authors' Information}

$\mathrm{OZ}$ is a Specialist Paramedic in Critical Care employed by South Western Ambulance Service NHS Foundation Trust (SWASFT) primarily deployed on a regional Helicopter Emergency Medical Service (HEMS). KK is employed clinically as a paramedic by SWASFT in addition to her main role as a senior research fellow with an interest in improving outcomes from out-of-hospital cardiac arrest, clinical decision-making and improving triage of emergency calls. MG is a lecturer in Paramedic Science on the undergraduate Paramedic Practice programme at Plymouth University in addition to his role supporting postgraduate students.

\section{References}

1. BHF, "Consensus Paper on Out-of-Hospital Cardiac Arrest in England," British Heart Foundation, London, 2015. 
2. OHCAO, "Out-of-Hospital Cardiac Arrest Outcomes Registry Epidemiology Report," Out of Hospital Cardiac Arrest Outcomes Project Team, Warwick, 2018.

3. J. Jentzer and C. Callaway, Cardiac Intensive Care, 3rd Edition ed., St Louis.: Elsevier, 2019.

4. S. Y. Lee, K. J. Hong, S. D. Shin, Y. S. Ro, K. J. Song, J. H. Park, S. Y. Kong, T. H. Kim and S. C. Lee, "The effect of dispatcher-assisted cardiopulmonary resuscitation on early defibrillation and return of spontaenous circulation with survival," Resuscitation, vol. 135, pp. 21-29, 2019.

5. J. Nolan, C. Deakin, J. Soar, G. Perkins, R. Davies, C. Thorne, Z. Fritz, S. Price, T. Keeble, M. Rowland, S. Shepherd, P. Dean, T. Billyard, A. Lockey, J. Wyllie and S. Hampshire, "Advanced Life Support 2021 Guidelines: Prehospital resuscitation," Resuscitation Council (UK), London, 2021.

6. J. Nolan, C. Sandroni, B. Bottiger, A. Cariou, T. Cronberg, H. Friberg, C. Genbrugge, K. Haywood, G. Lilja, V. Moulaert, N. Nikalaou, T. Olasveengen, M. Skrifvars, F. Taccone and J. Soar, “European Resuscitation Council and European Society of Intensive Care Medicine Guidelines 2021: PostResuscitation Care," Resuscitation, vol. 161, pp. 220-269, 2021.

7. C. Sinning, I. Ahrens, A. Cariou, F. Beygui, L. Lamhaut, S. Halvorsen, N. Nikalaou, J. Nolan, S. Price, K. Monsieurs, W. Behringer, M. Cecconi, E. Van Belle, X. Jouven and C. Hassager, "The cardiac arrest centre for the treatment of sudden cardiac arrest due to presumed cardiac cause - aims, function and structure: Position paper of the Association for Acute CardioVascular Care of the European Society of Cardiology (AVCV), European Assoc," European Heart Journal. Acute Cardiovascular Care, vol. 9, no. 4, pp. S193-S202, 2020.

8. K. Sunde, M. Pytte, D. Jacobsen, A. Mangschau, L. Jensen, C. Smedsrud, T. Draegni and P. Steen, "Implementation of a standardised treatment protocol for post resuscitation care after out-ofhospital cardiac arrest," Resuscitation, vol. 73, no. 1, pp. 29-39, 2007.

9. R. Knafelj, P. Radsel, T. Ploj and M. Noc, "Primary percutaneous coronary intervention and mild induced hypothermia in comatose survivors of ventricular fibrillation with ST-elevation acute myocardial infarction," Resuscitation, vol. 74, no. 2, pp. 227-234, 2007.

10. N. McKenzie, T. Williams, K. Ho, M. Inoue, P. Bailey, A. Celenza, D. Fatovich, I. Jenkins and J. Finn, "Direct transport to a PCl-capable hospital is associated with improved survival after adult out-ofhospital cardiac arrest of medical aetiology," Resuscitation, vol. 128, pp. 76-82, 2018.

11. D. Spaite, I. Stiell, B. Bobrow, M. de Boer, J. Maloney, K. Denninghoff, T. Vadeboncoeur, J. Dreyer and G. Wells, "Effect of Transport Interval on Out-of-Hospital Cardiac Arrest Survival in the OPALS Study: Implications for Triaging Patients to Specialized Cardiac Arrest Centers," Annals of Emergency Medicine, vol. 54, pp. 248-255, 2009.

12. V. Braun and V. Clarke, Successful Qualitative Research, London: Sage, 2013.

13. M. Gordon, C. Fell, H. Box, M. Farrell and A. Stewart, "Learning health 'safety' within non-technical skills interprofessional simulation education: a qualitative study," Med Educ Online, p. 22(1), 2017.

14. London Ambulance Service, "Advanced Clinical Operating Procedure: Sedation Post Cardiac Arrest," LAS, London, 2014. 
15. A. Cournoyer, E. Notebaert, L. de Montigny, D. Ross, S. Cossette, L. Londei-Leduc, M. Iseppon, Y. Lamarche, C. Sokoloff, B. Potter, A. Vadeboncoeur, D. Larose, J. Morris, R. Daoust, J.-M. Chauny, E. Piette, J. Paquet, Y. Cavayas, F. de Champlain, E. Segal, M. Albert and M.-C. Guertin, "Impact of the direct transfer to percutaneous coronary intervention-capable hospitals on survival to hsoptial discharge for patients with an out-of-hospital cardiac arrest.," Resuscitation, vol. 125, pp. 28-33, 2018.

16. G. Geri, J. Gilgan, W. Wu, S. Vijendira, C. Ziegler, I. Drennan, L. Morrison and S. Lin, "Does transport time of out-of-hospital cardiac arrest patients matter? A systemic review and meta-analysis," Resuscitation, vol. 115, pp. 96-101, 2017.

17. M. Cudnik, R. Schmicker, C. Vaillancourt, C. Newgard, J. Christenson, D. Davis and R. Lowe, "A geospatial assessment of transport distance and survival to discharge in out of hospital cardiac arrest patients: Implications for resuscitation centers.," Resuscitation, vol. 81, pp. 518-523, 2010.

18. K. Kajino, T. Iwami, M. Daya, T. Nsihuchi, Y. Hayashi, T. Kitamura, T. Irisawa, T. Sakai, Y. Kuwagata, A. Hiraide, M. Kishi and S. Yamayoshi, "Impact of transport to critical care medical centers on outcomes after out-of-hospital cardiac arrest.," Resuscitation, vol. 81, pp. 549-554, 2010.

19. R. Lyon and M. Nelson, "Helicopter emergency medical services (HEMS) response to out-of-hospital cardiac arrest," Resuscitation, vol. 83, p. e96, 2012.

20. T. Shimizu, I. Takahashi, S. Nara and Y. Morishita, "The usefulness of physician-led helicopter emergency medical," Resuscitation, vol. 83, pp. e23-e24, 2012.

21. J. von Vopelius-Feldt, A. Coulter and J. Benger, "The impact of a pre-hospital critical care team on survival from out-of-hospital cardiac arrest.," Resuscitation, vol. 96, pp. 290-295, 2015.

22. J. von Vopelius-Feldt, J. Powell and J. Benger, "Cost-effctiveness of advanced life support and prehospital critical care for out-of-hospital cardiac arrest in England: a descision analysis model.," BMJ Open, vol. 9, 2019.

23. HCPC, "Standards of proficiency: Paramedics," Health \& Care Professions Council, London, 2014.

24. A. Cournoyer, E. Notebaert, L. de Montigny, D. Ross, S. Cossette, L. Londei-Leduc, M. Iseppon, Y. Lamarche, C. Sokoloff, B. Potter, A. Vadeboncoeur, D. Larose, J. Morris, R. Daoust, J.-M. Chauny, E. Piette, J. Paquet, Y. Cavayas, F. de Champlain, E. Segal, M. Albert, M.-C. Guertin and A. Denault, "Impact of the direct transfer to percutaneous coronary intervention-capable hospitals on survival to hospital discharge for patients with out-of-hospital cardiac arrest," Resuscitation, vol. 125, pp. 28-33, 2018.

\section{Figures}




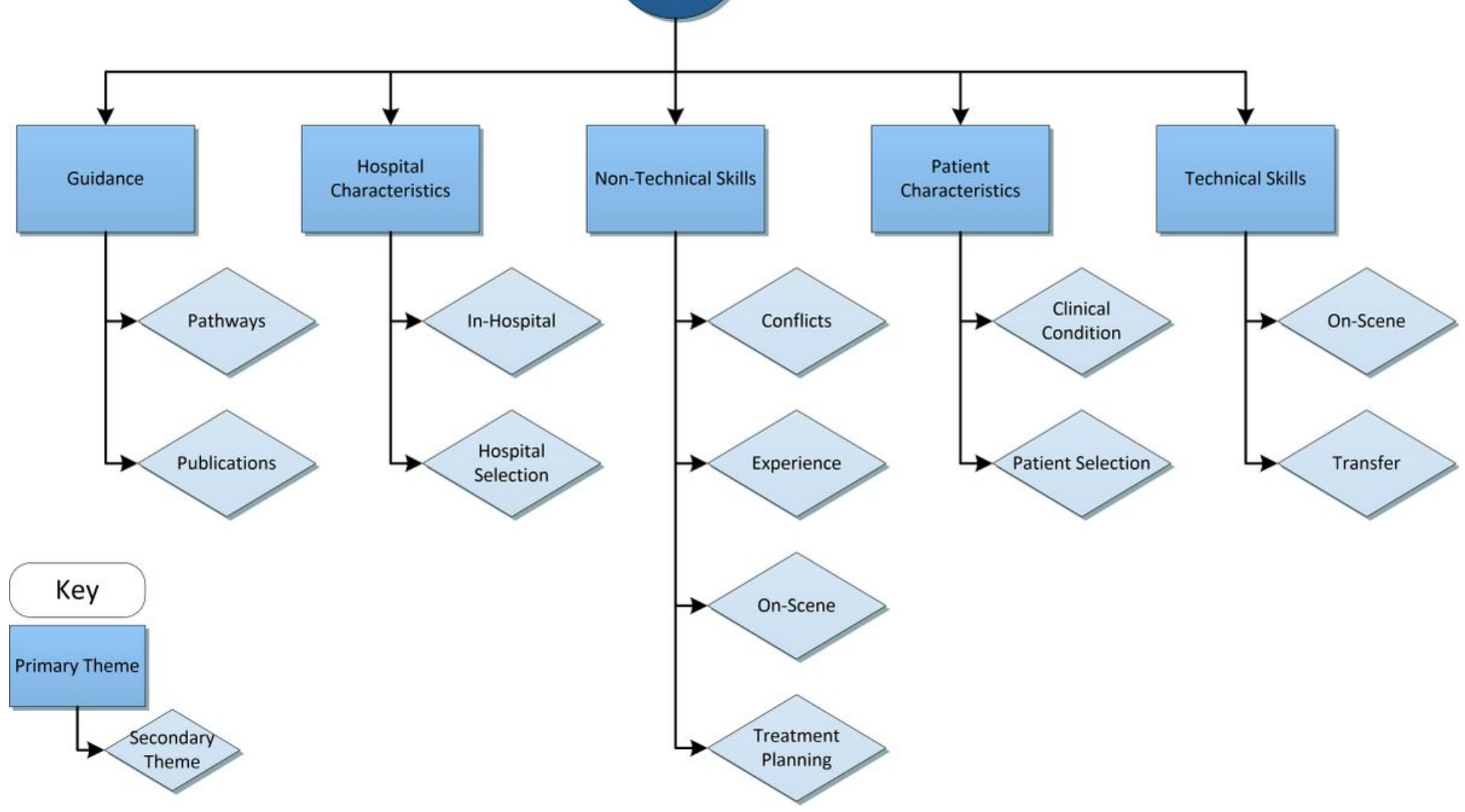

Figure 1

Thematic Map 